\title{
Research
}

\section{Mechanisms Affecting Population Density in Fragmented Habitat}

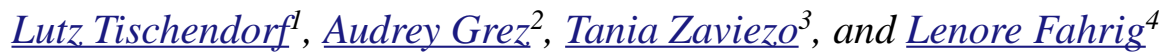

\begin{abstract}
We conducted a factorial simulation experiment to analyze the relative importance of movement pattern, boundary-crossing probability, and mortality in habitat and matrix on population density, and its dependency on habitat fragmentation, as well as inter-patch distance. We also examined how the initial response of a species to a fragmentation event may affect our observations of population density in post-fragmentation experiments. We found that the boundary-crossing probability from habitat to matrix, which partly determines the emigration rate, is the most important determinant for population density within habitat patches. The probability of crossing a boundary from matrix to habitat had a weaker, but positive, effect on population density. Movement behavior in habitat had a stronger effect on population density than movement behavior in matrix. Habitat fragmentation and inter-patch distance may have a positive or negative effect on population density. The direction of both effects depends on two factors. First, when the boundary-crossing probability from habitat to matrix is high, population density may decline with increasing habitat fragmentation. Conversely, for species with a high matrix-to-habitat boundary-crossing probability, population density may increase with increasing habitat fragmentation. Second, the initial distribution of individuals across the landscape: we found that habitat fragmentation and inter-patch distance were positively correlated with population density when individuals were distributed across matrix and habitat at the beginning of our simulation experiments. The direction of these relationships changed to negative when individuals were initially distributed across habitat only. Our findings imply that the speed of the initial response of organisms to habitat fragmentation events may determine the direction of observed relationships between habitat fragmentation and population density. The time scale of post-fragmentation studies must, therefore, be adjusted to match the pace of post-fragmentation movement responses.
\end{abstract}

Key Words: boundary crossing; emigration; habitat fragmentation; immigration; modeling; movement; population density; simulation; time scale

\section{INTRODUCTION}

It has been shown that habitat fragmentation, i.e., the breaking apart of habitat independent of habitat loss, may have a positive or negative effect on population density in remnant habitat patches (patch-size effects reviewed in Bowers and Matter 1997 and Bender et al. 1998; fragmentation effects reviewed in Debinski and Holt 2000 and Fahrig 2003). Habitat fragmentation may shift the balance between immigration and emigration through several mechanisms. Such shifts can produce negative (e.g., McGarigal and McComb 1995, Hovel and Lipcius 2001) and positive (e.g., McGarigal and McComb 1995, Collins and Barrett
1997, Collinge and Forman 1998, Dooley and Bowers 1998, Hovel and Lipcius 2001) effects of habitat fragmentation on population density or abundance.

First, increased habitat fragmentation may reduce population density. A higher perimeter:area ratio of habitat patches resulting from habitat fragmentation may increase the likelihood of organisms within patches encountering habitat-to-matrix boundaries, and thus result in a higher emigration rate. Emigrating organisms may also spend more time in the matrix, and may be subject to higher mortality, resulting in reduced population density. 
Second, increased habitat fragmentation can also increase population density. Habitat fragmentation reduces distances between remnant habitat patches (presuming that habitat amount and landscape extent remain constant), and increases habitat edge. More habitat edge may increase the likelihood that dispersing organisms will encounter a matrix-tohabitat boundary (e.g., Grez et al. in press). A larger number of smaller habitat patches may, therefore, "collect" more stranded individuals than a landscape composed of a smaller number of larger habitat patches (Fahrig 2003). Bowman et al. (2002) suggest that smaller patches will collect more individuals per unit area than larger patches for most conceivable movement behaviors.

Overall, whether habitat fragmentation increases or decreases, population density will depend on the balance of these mechanisms. Intuitively, it seems that this balance should depend on the movement behavior, particularly on the boundary-crossing behavior of the organism. To date, these arguments have not been formally and quantitatively addressed in the literature; the mechanisms and their interactions are still not fully understood (e.g., Sisk et al. 1997, Morales 2002, Brotons et al. 2003).

We addressed this situation by conducting an empirical habitat fragmentation experiment, accompanied by a simulation study. The intent of this complementary approach was to model observed movement behavior of four Coccinellid beetles in a fragmented alfalfa field (Grez et al. 2004), and to compare observed population density in habitat fragments with corresponding model predictions. During the course of our empirical experiment, we realized that the observed movement data were not sufficient to fully parameterize our movement model. Therefore, we decided to extend our simulation experiment by conducting a factorial combination of behavioral movement parameters in a quest to explain their effect and relative importance on the relationships between habitat fragmentation, inter-patch distance, and population density.

The following paper presents the results of our simulation study. Our work addressed the following research questions:

1. How do behavioral characteristics, such as movement pattern (velocity and directionality), mortality, and boundary-crossing probability from habitat to matrix and from matrix to habitat affect population density in habitat?

2. What is the relative importance of each of these behavioral characteristics?

3. How do these behavioral characteristics influence the relationships between population density and both habitat fragmentation and inter-patch distance?

4. How does the initial distribution of organisms in short-term, post-fragmentation experiments affect the relationship between habitat fragmentation and inter-patch distance and population density?

To our knowledge, this is the first attempt to quantify the relative importance of behavioral characteristics of moving individuals on population density, and its dependency on habitat fragmentation as well as inter-patch distance. We are also not aware of any other study explicitly analyzing the effect of the initial spatial distribution of organisms in post-fragmentation experiments. As our results reveal, the boundary-crossing probability of organisms and their immediate response to fragmentation events may determine the observed relationship between habitat fragmentation and population density in short-term, postfragmentation studies.

\section{METHODS}

\section{Model Landscapes}

We developed a computer model to simulate movement, boundary crossing, and mortality of individuals across binary landscapes comprising habitat and matrix. We created four model landscapes on a $30 \times 30$ square-cell grid (Fig. 1). The configuration of habitat in the four model landscapes corresponds to the layout of our empirical habitat fragmentation experiment (Grez et al. 2004). Each cell represents one square meter of the experimental landscapes. Of all the 900 cells, $16 \%$ or 144 cells were designated as habitat. The remaining $84 \%$ of all cells were defined as matrix. The borders of the model landscapes were open to moving individuals. If an individual left the model landscape, it continued moving in an infinite virtual space surrounding the model landscape until it died or re-entered the model landscape. This assumption is realistic with respect to insects (our study species), 
which may leave and re-enter experimental landscapes.

\section{Movement Pattern}

We modeled movement as a correlated random walk. Each movement step was defined by a vector between two consecutive locations (see also Tischendorf and Fahrig 2000, Tischendorf 2001). The new location was calculated based on a random step length and a random step angle. The random step length (H_STP_L or M_STP_L, Table 1) was drawn from a negative exponential distribution. The random step angle was drawn from a uniform distribution between 0 and 360 degrees (H_STP_A or M_STP_A, Table 1). Both step length and step angle were set separately for habitat and matrix. This approach allows simulation of different movement patterns on habitat and matrix.

The step length was adjusted to the simulation time (50 time or movement steps) so that the maximum possible movement distance was close to the landscape model extent (30 cells). The maximum possible movement distance varied also with variations in step angle and boundary-crossing rates and did not always match the landscape extent exactly. However, the scale of movement and landscape were generally adjusted.

\section{Boundary Crossing}

The two landcover types, habitat and matrix, result in two boundary types: habitat-to-matrix and matrix-to-habitat (H_M_CROSS or M_H_CROSS, Table 1). We defined the probability of one individual crossing each boundary type by two boundary-crossing parameters, which could be set independently from each other. If an individual did not cross a boundary, the movement direction was reversed and its position was set to its previous location.

\section{Mortality}

We modeled mortality as the probability of an individual dying during one time step. Mortality was set separately for habitat and matrix (H_MORT or M_MORT, Table 1), with a range of mortality rates lower in habitat than in matrix reflecting that the latter is poorer environment. Mortality parameter values were set such that up to $30 \%$ of the initial population could die during 50 simulation time steps. This assumption reflects severe effects of mortality on population density in short-term, postfragmentation studies, and may overestimate real mortality. However, it allowed us to rank mortality based on a strong effect on population abundance.

\section{Initial Distribution}

We initialized our computer model in two different ways. At the beginning of each simulation run, 1000 individuals were either distributed uniformly across the entire model landscape (i.e., across habitat and matrix, LAND_INIT, Table 1) or across habitat area only (HAB_INIT, Table 1). These two initial distributions reflect the potential immediate response of organisms to fragmentation events, such as ploughing, mowing, or forest clearcutting. LAND_INIT is more likely to represent an appropriate initialization for ground-dwelling species, such as beetles, spiders, or plants, which are more likely to stay in the matrix during the fragmentation process, for example forest cutting. These species are likely to be found in the matrix shortly after a fragmentation event. Their movement into habitat may be delayed. HAB_INIT, on the other side, is more likely to represent flying species in general, which may move into habitat remnants during the fragmentation process. These species will likely not be found in the matrix shortly after a fragmentation event.

\section{Simulation Experiment}

At the beginning of each simulation run, 1000 individuals were randomly distributed across the entire model landscape (LAND_INIT) or within habitat (HAB_INIT). We simulated 50 movement steps and recorded population density within habitat at the end of each simulation run. We chose 50 movement steps, because the maximum observed movement distance corresponded to the landscape extent. Our results are principally invariant to this assumption, because population abundance declined linearly with increasing simulation time without changes in the relative importance of the model variables.

The simulation experiment consisted of 6561 factorial combinations of the movement, boundary- 
Fig. 1. Model landscapes used in the simulation model. Black areas are habitat and white areas are matrix. The model landscapes represent two levels of fragmentation (4 vs. 16 habitat patches) and two levels of inter-patch distance (2-cell vs. 6-cell gaps between habitat patches).
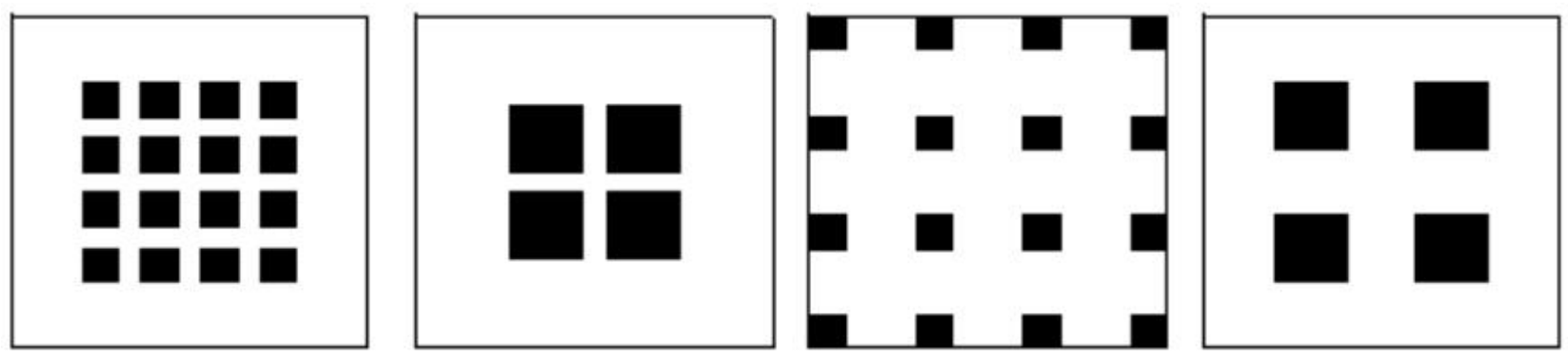

Table 1. Parameter values used in the factorial simulation experiment

\begin{tabular}{|c|c|c|c|}
\hline Explanation (unit) & Parameter name & Parameter values & Levels \\
\hline $\begin{array}{l}\text { Step length on habitat (mean of negative } \\
\text { exponential distribution, cells) }\end{array}$ & H_STP_L & $0.3,0.39,0.55$ & 3 \\
\hline Step angle on habitat (cells) & H_STP_A & $+/-45^{\circ},+/-70^{\circ},+/-90^{\circ}$ & 3 \\
\hline $\begin{array}{l}\text { Step length on matrix (mean of negative } \\
\text { exponential distribution, cells) }\end{array}$ & M_STP_L & $0.3,0.39,0.55$ & 3 \\
\hline Step angle on matrix (cells) & M_STP_A & $+/-45^{\circ},+/-70^{\circ},+/-90^{\circ}$ & 3 \\
\hline $\begin{array}{l}\text { Boundary-crossing probability per encounter from } \\
\text { habitat to matrix (percentage) }\end{array}$ & H_M_CROSS & $10,50,90$ & 3 \\
\hline $\begin{array}{l}\text { Boundary-crossing probability per encounter from } \\
\text { matrix to habitat (percentage) }\end{array}$ & M_H_CROSS & $10,50,90$ & 3 \\
\hline $\begin{array}{l}\text { Mortality probability per individual per time step } \\
\text { on habitat }\end{array}$ & H_MORT & $0.001,0.003,0.005$ & 3 \\
\hline $\begin{array}{l}\text { Mortality probability per individual per time step } \\
\text { on matrix }\end{array}$ & M_MORT & $0.005,0.007,0.009$ & 3 \\
\hline Initialization & INIT & LAND_INIT, HAB_INIT & 2 \\
\hline Fragmentation (\# fragments) & FRAG & 4,16 & 2 \\
\hline Inter-patch distance (cells) & GAP & 2,6 & 2 \\
\hline
\end{tabular}


crossing, and mortality parameter values in Table 1. This simulation experiment was repeated for each initial distribution (LAND_INIT and HAB_INIT, see above) and on each of the four model landscapes. The total number of unique parameter combinations was, therefore, 52488 (6561*2*4), and each combination was replicated 50 times, resulting in 2 624400 simulation runs.

\section{Data Analysis}

We first determined the main effects and relative importance of each model parameter on population density within habitat (based on Type III SS). Then, we analyzed main and interaction effects of a subset of the most important model parameters on population density using a two-way ANOVA (SAS Institute 1990).

\section{RESULTS}

\section{Overview}

Population density was primarily affected by boundary-crossing probabilities from habitat to matrix and from matrix to habitat (see main effects of H_M_CROSS and M_H_CROSS in Table 2, Fig. $2 \mathrm{a}, \overline{\mathrm{b}})$. Main effects of habitat fragmentation and inter-patch distance on population density were negative (FRAG and GAP in Table 2, Fig. 2c, d). However, the direction of the effects of habitat fragmentation and inter-patch distance on population density depended on the initial distribution of individuals and on the boundarycrossing probability (Table 3 ). When individuals were initially distributed across habitat and matrix, habitat fragmentation and inter-patch distance showed positive relationships with population density. Conversely, initial distribution of individuals across habitat only resulted in negative relationships between habitat fragmentation, interpatch distance, and population density (FRAG* INIT and GAP*INIT in Table 3, Fig. 3a, b). Also, a low boundary-crossing probability from habitat to matrix resulted in a positive effect of habitat fragmentation on population density (FRAG* H_M_CROSS in Table 3, H_M_CROSS = 10 in Fig. 4 a). Conversely, a high boundary-crossing probability from habitat to matrix resulted in a negative effect of habitat fragmentation on population density (H_M_CROSS = 90 in Fig. 4a).

\section{Effects of Movement Pattern, Mortality, and Boundary-crossing Probability}

Population density was negatively related to movement step length on habitat and positively correlated to movement step angle on habitat (H_STP_L and H_STP_A, Table 2). Faster and more directed movement patterns on habitat increase the probability of habitat-to-matrix boundary encounters (and, therefore, the likelihood of emigration), resulting in a negative effect on population density within habitat patches. Movement step length on matrix had a positive effect on population density, whereas increased movement step angles on matrix decreased population density (M_STP_L and M_STP_A, Table 2). Faster movement and more directed movement paths on matrix increase the probability of matrix-to-habitat boundary encounters (and, therefore, the likelihood of immigration) resulting in a positive effect on population density in habitat. Mortality in both habitat and matrix reduced population density (H_MORT and M_MORT, Table 2). As noted above, boundary-crossing probability from habitat to matrix was negatively correlated to population density (H_M_CROSS, Table 2 ). In contrast, boundary-crossing probability from matrix to habitat increased population density in habitat (M_H_CROSS, Table 2).

\section{Relative Importance of Behavioral Characteristics}

The most important determinant for population density was the boundary-crossing probability from habitat to matrix (H_M_CROSS, Table 2 and Fig. 2a). The boundary-crossing probability from matrix to habitat ranked second, with a positive effect on population density (M_H_CROSS, Table 2 and Fig. 2b). Movement pattern and mortality in habitat were generally more important for population density than movement pattern and mortality in matrix. Movement step length (i.e., velocity) ranked before mortality and movement step angle (i.e., directionality). Overall, boundary-crossing probabilities from habitat to matrix and vice versa had the strongest effects on population density, followed by movement pattern and mortality in habitat. Movement behavior and mortality in matrix were least important for population density in habitat. 
Table 2. Main effects between independent variables and population density. Type III sums of square values indicate the relative importance of the main effects

\begin{tabular}{llll}
\hline \hline Independent Variables & DF & $\begin{array}{l}\text { Type III Sums of } \\
\text { Squares }\end{array}$ & Direction \\
\hline H_M_CROSS & 2 & 230.718953 & - \\
INIT & 1 & 214.059342 & $\mathrm{n} / \mathrm{a}$ \\
M_H_CROSS & 2 & 69.9165933 & + \\
H_STP_L & 2 & 29.3735549 & - \\
FRAG & 1 & 23.6006651 & - \\
H_STP_A & 2 & 13.8723842 & + \\
H_MORT & 2 & 5.8036627 & - \\
M_STP_L & 2 & 5.5992344 & + \\
GAP & 1 & 5.2671948 & - \\
M_MORT & 2 & 0.6042428 & - \\
M_STP_A & 2 & 0.0373473 & - \\
\hline
\end{tabular}

\section{Interaction between Behavior, Habitat Fragmentation, and Inter-patch Distance}

The magnitude and direction of the effect of habitat fragmentation on population density depended in part on the boundary-crossing probability from habitat to matrix and from matrix to habitat. A low boundary-crossing probability from habitat to matrix resulted in a positive effect of habitat fragmentation on population density, whereas a high boundary-crossing probability from habitat to matrix resulted in a negative effect (see FRAG* H_M_CROSS, Table 3 and Fig. 4a). Conversely, a high boundary-crossing probability from matrix to habitat resulted in a positive effect of habitat fragmentation on population density (see FRAG* M_H_CROSS, Table 3 and Fig. 4b). This effect was negative, when boundary-crossing probability from matrix to habitat was low. The effect of inter-patch distance on population density was equally affected by boundary-crossing probabilities (see GAP* H_M_CROSS and GAP*M_H_CROSS, Table 3 and Fig. 4c, d). Movement step length, step angle, and mortality did not interact significantly with habitat fragmentation and inter-patch distance on population density.

\section{Interaction between Initial Distribution, Habitat Fragmentation, and Inter-patch Distance}

Our results revealed strong interaction effects between the initial distribution and habitat fragmentation (see FRAG*INIT in Table 3 and Fig. $3 \mathrm{a})$ and between the initial distribution and interpatch distance (see GAP*INIT in Table 3 and Fig. $3 b)$. Both interaction effects indicate that the initial distribution of individuals can determine the direction of the observed relationship between either habitat fragmentation or inter-patch distance and population density. If individuals are randomly distributed across habitat and matrix shortly after a fragmentation event, observers may actually perceive higher population densities in more fragmented habitat in post-fragmentation studies. However, if organisms react immediately, and are found only within habitat fragments shortly after a fragmentation event, observers may perceive lower population density in more fragmented habitat. The relationship between inter-patch distance shows a similar dependency from the initial distribution (Fig. 3b). 
Fig. 2. Main effects of boundary-crossing probabilities, initial distribution of individuals, habitat fragmentation, and inter-patch distance on population density in habitat.

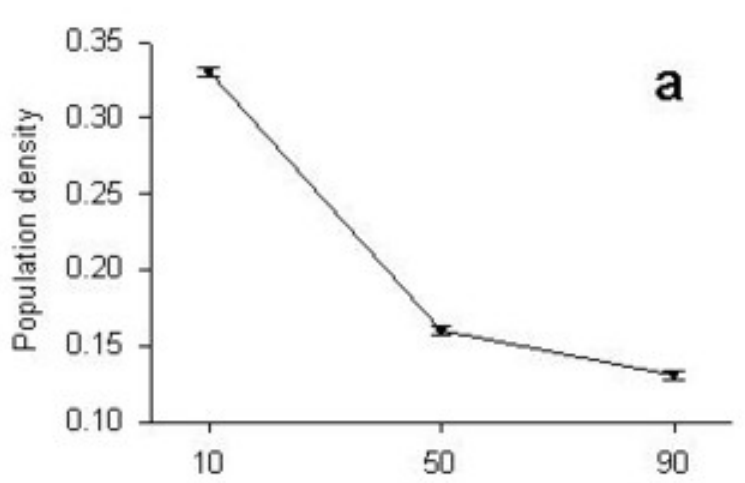

Boundary crossing probability from habitat to matrix $\%$ (H_M_CROSS)
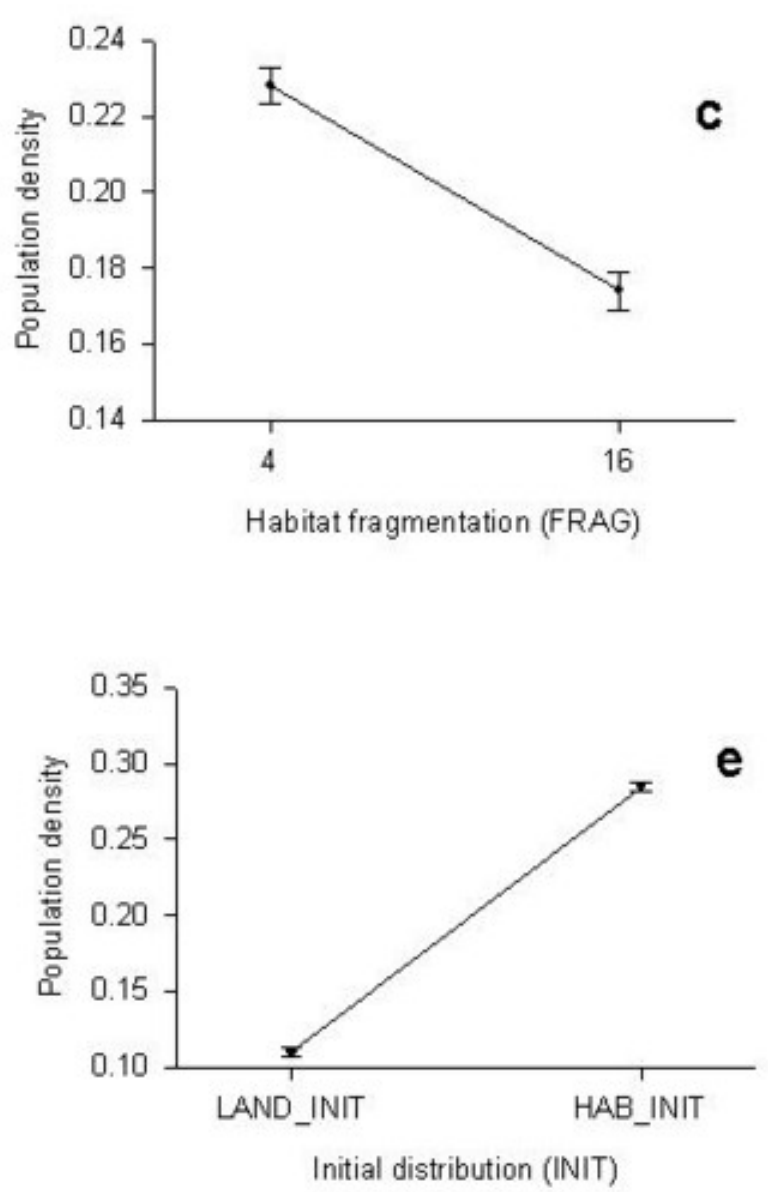

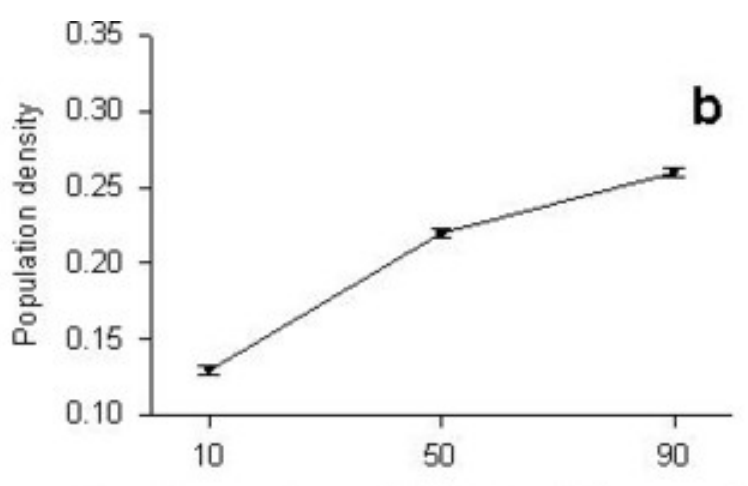

Boundary crossing probability from habitat to matrix \% (M_H_CROSS)

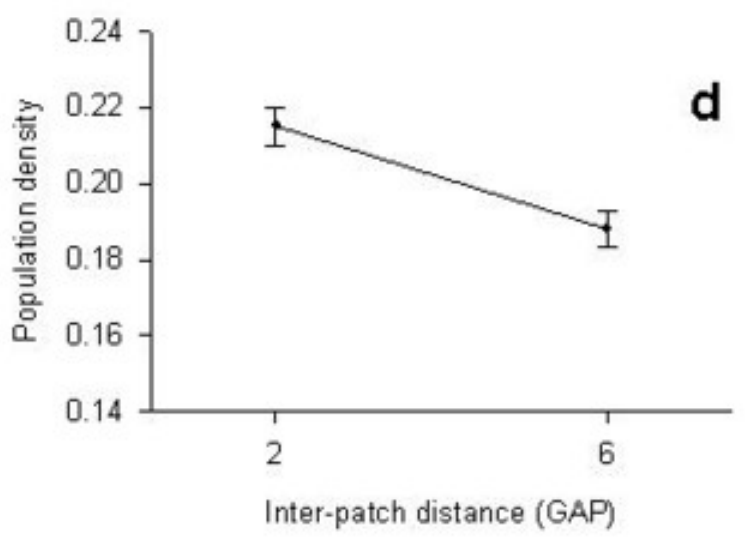


Table 3. Interaction effects between independent variables and population density. Type III sums of square values indicate the relative importance of the interaction effects

\begin{tabular}{lll}
\hline \hline Independent Variables & DF & Type III Sums of Squares \\
\hline FRAG*INIT & 1 & 30.115648 \\
GAP*INIT & 1 & 7.6483550 \\
GAP*M_H_CROSS & 2 & 0.5123123 \\
FRAG*H_M_CROSS & 2 & 0.4183648 \\
GAP*H_M_CROSS & 2 & 0.2589531 \\
FRAG*M_H_CROSS & 2 & 0.1702674 \\
\hline
\end{tabular}

\section{DISCUSSION}

The results of our simulation study revealed mechanisms for some of the hypotheses, which were used to explain the inconsistency in observed relationships between habitat fragmentation and population density (see Bowman et al. 2002, Fahrig 2003). The most interesting findings of our study are the relative importance of and interaction effects between behavioral characteristics and habitat fragmentation on population density.

The most important determinant for population density in the remaining habitat patches was the boundary-crossing probability from habitat to matrix. This finding confirms our intuition that a higher boundary-crossing probability from habitat to matrix increases the emigration rate and, therefore, reduces population density in habitat. It is less obvious, however, that the boundary-crossing probability from matrix to habitat, which determines the immigration rate, had a much weaker effect on population density. Yet, we varied both boundary-crossing parameter values across the same range. The difference in the strength of the effects can be explained by the perimeter:area (p:a) ratio of matrix and habitat. The p:a ratio for habitat is 1.33 in the model landscapes containing 16 habitat fragments and 0.66 in those containing four habitat fragments. In contrast, the p:a ratio for matrix is 0.25 and 0.127 in the model landscapes with 16 and four habitat fragments, respectively. The p:a ratio is positively correlated with the probability of boundary encounter (Fagan et al. 1999). In fact, we found a linear relationship between the probability of boundary encounters and p:a ratio, which is robust against variations in movement velocity and directionality (unpublished results, see also Bevers and Flather 1999). Individuals residing in the matrix will, therefore, have a much lower chance to encounter a boundary to habitat than those residing within habitat. This lower chance translates into a lower boundary-crossing frequency, which is the basis for the boundary-crossing probability from matrix to habitat. Therefore, immigration may have a weaker effect on population density than emigration in landscapes containing less habitat than matrix (see also Krauss et al. (2003) for relationships between habitat area and immigration rates, and Buechner (1987) for other factors affecting boundary-crossing probabilities).

Population density was also strongly affected by movement velocity, directionality, and mortality in habitat. In fact, all behavioral variables on habitat had a stronger effect on population density than their counterparts on matrix. Higher movement velocity and directionality on habitat resulted in lower population density. This effect is caused by the probability of boundary encounters, which increases with movement velocity and directionality. The overall magnitude of these effects is higher for habitat than for matrix-related movement behavior, because of the generally lower p:a ratio for matrixto-habitat boundaries in landscapes with less habitat than matrix.

Our results revealed interaction effects, which may explain why population density is sometimes found to be positively or negatively related to habitat 
Fig. 3. Interaction effects between initial distribution, habitat fragmentation, and inter-patch distance on population density in habitat.

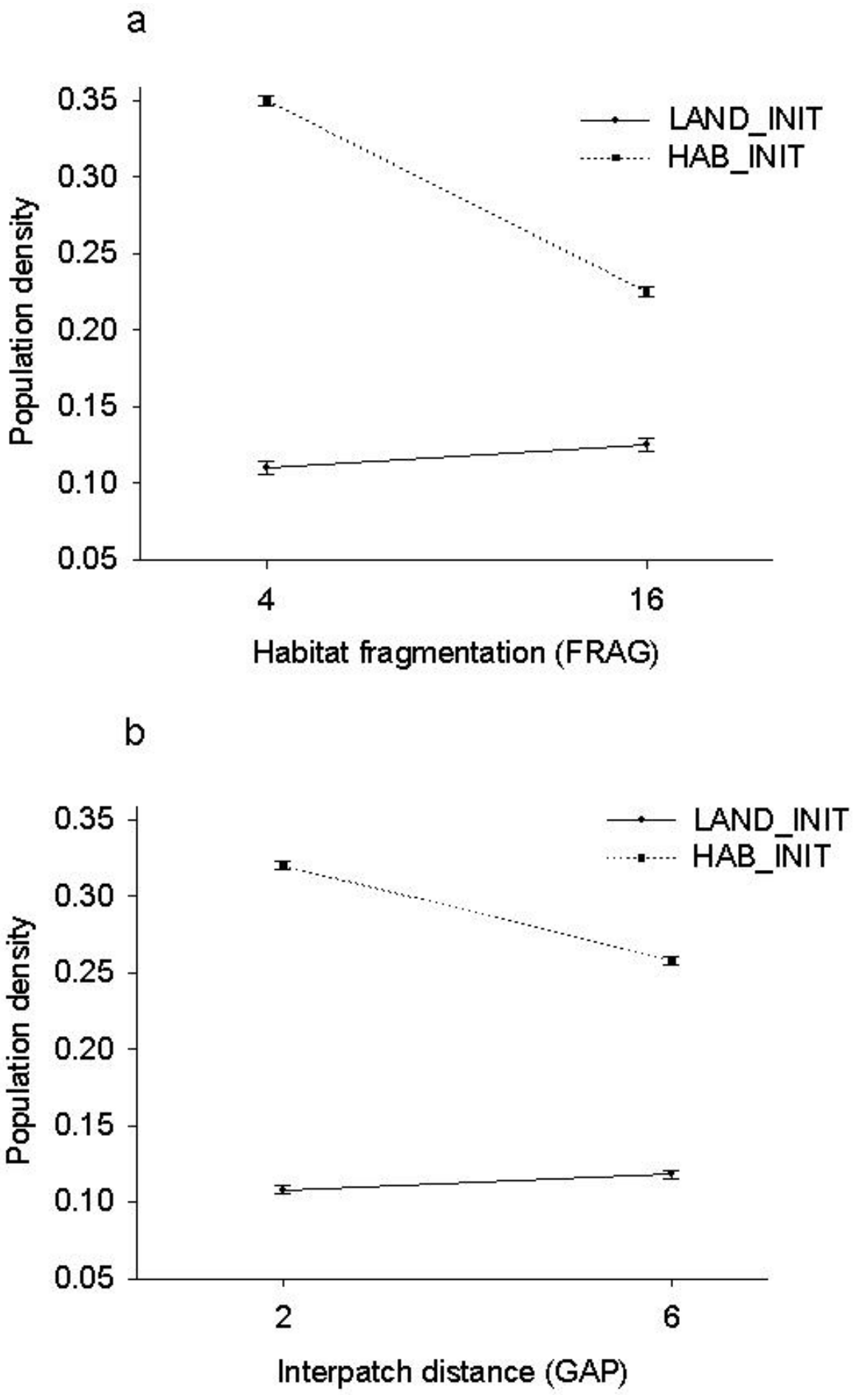


Fig. 4. Interaction effects between boundary-crossing probabilities, habitat fragmentation, and inter-patch distance on population density in habitat.
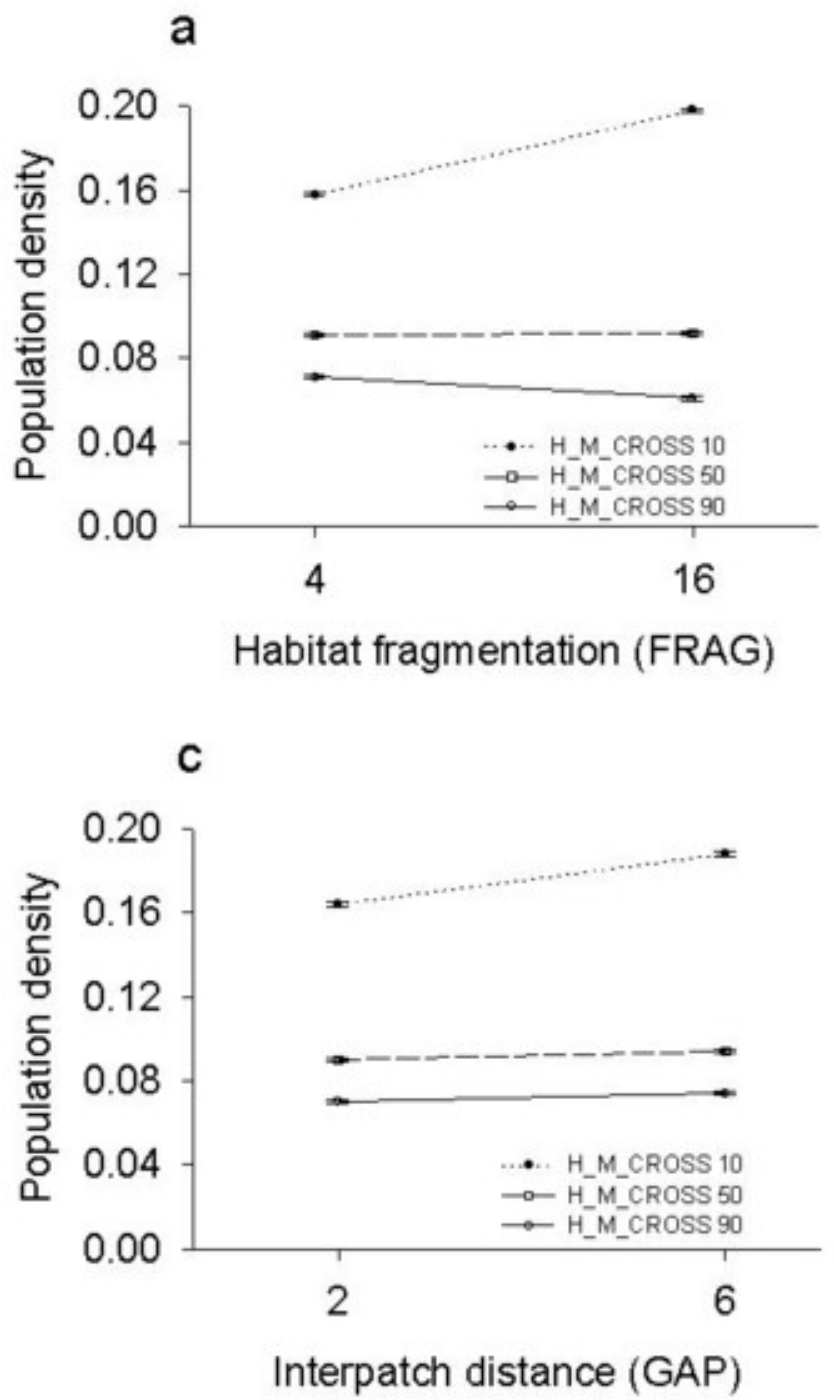
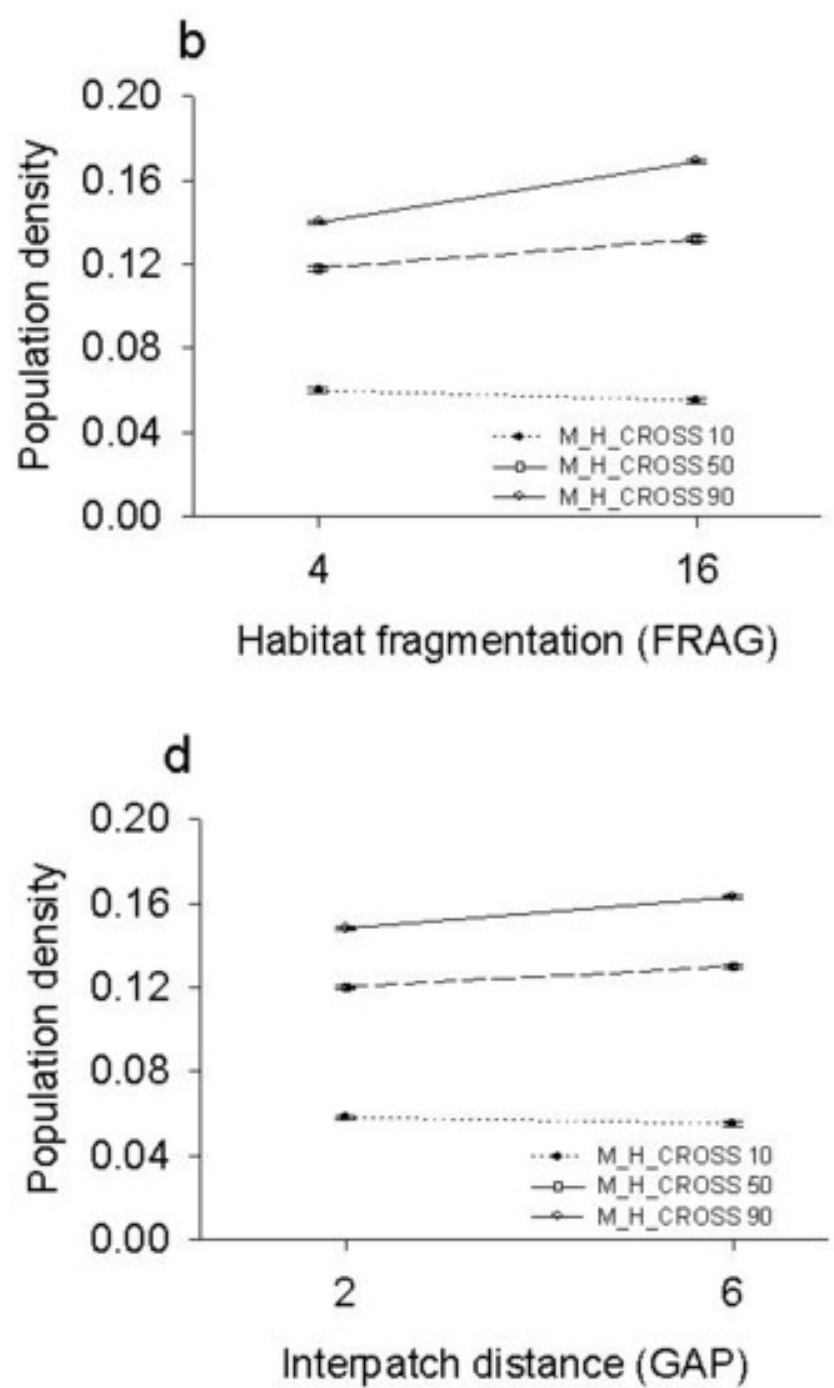

fragmentation and inter-patch distance. As we suggested in the Introduction, this balance should depend on the movement behavior, particularly the boundary-crossing behavior of the organism. We found significant interaction effects between boundary-crossing probabilities and habitat fragmentation, as well as inter-patch distance on population density. All other movement variables did not show such interaction effects on population density. This suggests that boundary-crossing behavior (i.e., reluctance or eagerness to cross a boundary between habitat and matrix or vice versa) may determine how we perceive the relationship between habitat fragmentation and population density. For habitat specialists, which may be more reluctant to leave habitat, fragmentation may result in higher population densities. This effect is likely to be transient, because a lower emigration rate will ultimately reduce the probability of recolonizing habitat fragments after local extinctions. Conversely, 
for generalist species, which are more willing to leave habitat, population density may decline with increasing habitat fragmentation (Bommarco and Fagan 2002).

Furthermore, we found the direction and magnitude of the effects of habitat fragmentation and interpatch distance on population density to be dependent on the initial distribution of individuals in the model landscapes. This finding has implications for short-term, post-fragmentation studies. Most empirical studies examining postfragmentation effects on population dynamics, and population density in particular, are short-term studies conducted immediately or shortly after fragmentation events. In fact, our empirical habitat fragmentation experiment was conducted 3 weeks before and up to 12 weeks after ploughing the matrix in an alfalfa field. An examination of the initial response or distribution of a study species after a fragmentation event may, therefore, be crucial to the observed change in population density in postfragmentation studies. Flying species are likely to respond immediately to ploughing, mowing, or clearcutting. Ground-dwelling species, such as small mammals, some spiders, or plants, may respond much more slowly and result in a different observation of population density. It is, therefore, necessary to not just account for the initial response, but also to adjust the time scale of postfragmentation studies to the speed at which species are likely to adjust to changes in their habitat configuration. In the case of insects, weeks might be an appropriate time scale, whereas other species may respond over a time period of years. Our observations of population density will, therefore, not just depend on habitat fragmentation or interpatch distance, but also on the time period of the study after a fragmentation event. Our finding also suggests that some observed effects may be transient and may even turn over when considered at a larger time scale (see also McGarigal and McComb 1995, Flather et al. 1999).

Finally, we are aware that our results may not apply to all conceivable and observed movement behaviors. Future work may relate the results of this study to density-dependent emigration or immigration rates (e.g., Andreassen and Ims 2001), as well as movement orientation (Zollner and Lima 1997, 1999a, 1999b) and more systematic movement strategies (e.g., Conradt et al. 2003, Russell et al. 2003). Some species may also change boundary-crossing behavior throughout the year
(Bommarco and Fargan 2002), depending on habitat quality (Lin and Batzli 2001, 2004) or through interactions with other species (Fagan et al. 1999).

\section{CONCLUSION}

The results of our study confirmed that effects of habitat fragmentation and inter-patch distances on population density may indeed be positive or negative. The magnitude and direction of these effects is primarily affected by boundary-crossing probabilities from habitat to matrix and vice versa, and by the initial response of a species to habitat fragmentation. From our study, we draw the following conclusions:

1. Movement behavior in habitat has a stronger effect on population density than movement behavior in matrix, when there is less habitat than matrix in a landscape.

2. Boundary-crossing probability from habitat to matrix had the strongest negative effect on population density.

3. Boundary-crossing probability from matrix to habitat had the strongest positive effect on population density.

4. Emigration may have a stronger effect on population density than immigration, because of a higher p:a ratio for habitat than matrix in fragmented landscapes with less habitat than matrix.

5. Increasing habitat fragmentation and interpatch distance may result in increased observed population density when boundarycrossing probability from habitat to matrix is low (e.g., habitat specialist species), or when species respond slowly to habitat fragmentation events.

6. In contrast to conclusion 5 , increasing habitat fragmentation and inter-patch distance may result in lower observed population density when boundary-crossing probability from matrix to habitat is low (e.g., habitat generalist species), or when species respond immediately to habitat fragmentation events.

7. Post-fragmentation studies should be 
adjusted to the time scale of a species' response to fragmentation events, because fragmentation effects on population density may be transient and change over time.

Responses to this article can be read online at: http://www.ecologyandsociety.org/vollo/iss 1/art7/responses/

\section{Acknowledgments:}

This work was funded in part by the Chilean government through Fondecyt 1011041 and 7011041 and by ELUTIS Modelling and Consulting Inc.

\section{LITERATURE CITED}

Andreassen, H. P., and R. A. Ims. 2001. Dispersal in patchy vole populations: role of patch configuration, density dependence, and demography. Ecology 82:2911-2926.

Bender, D. J., T. A. Contreras, and L. Fahrig. 1998. Habitat loss and population decline: a metaanalysis of the patch size effect. Ecology 79:517533.

Bevers, M., and C. H. Flather. 1999. Numerically exploring habitat fragmentation effects on population using cell-based coupled map lattices. Theoretical Population Biology 55:61-76.

Bommarco R., and W. F. Fagan. 2002. Influence of crop edges on movement of generalist predators: a diffusion approach. Agricultural and Forest Entomology 4:21-30

Bowers, M. A., and S. F. Matter. 1997. Landscape ecology of mammals: relationships between density and patch size. Journal of Mammalogy 78:9991013.

Bowman, J., N. Cappuccino, and L. Fahrig. 2002. Patch size and population density: the effect of immigration behavior. Conservation Ecology 6:9 (Online.) URL:

http://www.consecol.org/vol6/iss1/art9.
Brotons, L., M. Moenkkoenen, and J. L. Martin. 2003. Are fragments islands? Landscape context and density - area relationships in boreal forest birds. American Naturalist 162:343-357.

Buechner, M. 1987. Conservation in insular parks: simulation models of factors affecting the movement of animals across park boundaries. Biological Conservation 41:57-76.

Collins, R. J., and G. W. Barrett. 1997. Effects of habitat fragmentation on meadow vole (Microtus pennsylvanicus) population dynamics in experiment landscape patches. Landscape Ecology 12:63-76.

Collinge, S. K., and R. T.T . Forman. 1998. A conceptual model of land conversion processes: predictions and evidence from a microlandscape experiment with grassland insects. Oikos 82:66-84.

Conradt, L., P. A. Zollner, T. J. Roper, K. Frank, and C.D.Thomas. 2003. Foray search: an effective systematic dispersal strategy in fragmented landscapes. American Naturalist 161:905-915.

Debinski, D. M., and R. D. Holt. 2000. A survey and overview of habitat fragmentation experiments. Conservation Biology 14:342-355.

Dooley, J. L. and M. A. Bowers. 1998. Demographic responses to habitat fragmentation: experimental tests at a landscape and patch scale. Ecology 79:969-980.

Fagan, W. F., R. S. Cantrel, and C. Cosner. 1999: How habitat edges can change species interactions. American Naturalist 153:165-182.

Fahrig, L. 2003. Effects of habitat fragmentation on biodiversity. Annual Review of Ecology, Evolution, and Systematics 34:487-515.

Flather, C. H., M. Bevers, E. Cam, J. Nichols, and J. Sauer. 1999. Habitat arrangement and extinction thresholds: do forest birds conform to model predictions? Pages 44-45 in Landscape ecology: the science and the action, Vol. 1. $5^{\text {th }}$ World Congress, International Association for Landscape Ecology, Snowmass, Colorado, USA.

Grez, A., T. Zaviezo, and M. Rios. 2005. Ladybird (Coleoptera: Coccinellidae) dispersal in fragmented alfalfa landscapes. European Journal of Entomology: 
in press.

Grez, A., T. Zaviezo, L. Tischendorf, and L. Fahrig. 2004. A transient, positive effect of habitat fragmentation on insect population densities. Oecologia 141:444-451

Hovel, K. A., and R. N. Lipcius. 2001. Habitat fragmentation in a seagrass landscape: patch size and complexity control blue crab survival. Ecology 82:1814-29

Krauss, J., I. Steffan-Dewenter, and T. Tscharntke. 2003. Local species immigration, extinction, and turnover of butterflies in relation to habitat area and habitat isolation. Oecologia 442:591-602.

Lin, Y. K., and K. O. Batzli. 2001. The influence of habitat quality on dispersal, demography, and population dynamics of voles. Ecological Monographs 71:245-275.

Lin, Y. K., and K. O. Batzli. 2004. Movement of voles across habitat boundaries: effects of food and cover. Journal of Mammalogy 85:216-224.

McGarigal, K., and W. C. McComb. 1995. Relationships between landscape structure and breeding birds in the Oregon Coast Range. Ecological Monographs 65:235-260.

Morales, J. M. 2002. Behavior at habitat boundaries can produce leptokurtic movement distributions. The American Naturalist 160:531538.

Russell, R. E., R. K. Swihart, and Z. Feng. 2003. Population consequences of movement decisions in a patchy landscape. Oikos 103:142-152.

SAS Institute. 1990. SAS Version 6. SAS Institute, Cary, North Carolina, USA.

Sisk, T. D., N. M. Haddad, and P. R. Ehrlich. 1997. Bird assemblages in patchy woodlands: modeling the effects of edge and matrix habitats. Ecological Applications 7:1170-1180.

Tischendorf, L. 2001. Can landscape indices predict ecological processes consistently? Landscape Ecology 16:235-254.

Tischendorf, L., and L. Fahrig. 2000. How should we measure landscape connectivity? Landscape Ecology 15:633-641.

Zollner, P. A., and S. L. Lima. 1997. Landscapelevel perceptual abilities in white-footed mice: perceptual range and the detection of forest habitat. Oikos 80:51-60.

Zollner, P.A., and S. L. Lima. 1999a. Orientational data and perceptual range: real mice aren't blind. Oikos 84:164-166.

Zollner, P. A., and S. L. Lima. 1999b. Search strategies for landscape-level inter-patch movements. Ecology 80:1019-1030. 